\title{
Geociências
}

\section{Valores de background geoquímico e suas implicações em estudos ambientais}

\author{
(Geochemical background values and its implications in \\ environmental studies)
}

\begin{abstract}
Aline Sueli de Lima Rodrigues
Engenheira Ambiental, Msc. em Ciências Naturais e Doutoranda do Programa de Pós-Graduação em Evolução Crustal e Recursos Naturais, Laboratório de Geoquímica Ambiental ( $L G q A)$, Departamento de Geologia, Universidade Federal de Ouro Preto (UFOP), MG.E-mail: aline@degeo.ufop.br

Hermínio Arias Nalini Júnior

Professor, Doutor do Departamento de Geologia e do Programa de Pós-Graduação em Evolução Crustal e Recursos Naturais, Laboratório de Geoquímica Ambiental ( $L G q A)$, Universidade Federal de Ouro Preto

(UFOP), MG.E-mail: nalini@degeo.ufop.br
\end{abstract}

\section{Resumo}

O estabelecimento de valores de background geoquímico como medida relativa para distinguir concentrações naturais de um elemento (geogênica e/ou biogênica) e a influência das atividades antrópicas nestas concentrações representa um dos assuntos mais importantes das ciências ambientais recentes. A relação entre as alterações naturais e as proporcionadas pela ação antrópica nas espécies químicas é uma questão que envolve implicações importantes nas áreas da geologia, toxicologia e biologia, entre outros campos do conhecimento. Isto é especialmente importante, quando interpretações geoquímicas de elementos tóxicos são requeridas. Assim, o objetivo principal desse artigo é apresentar e discutir as razões pelas quais o estabelecimento de valores de background geoquímico tem implicações nos estudos ambientais. Ao longo desse artigo, são apresentados alguns conceitos de background geoquímico e os principais métodos utilizados para avaliar a concentração background de elementos químicos (métodos direto e indireto). Além disso, são destacados alguns exemplos de estudos desenvolvidos no Brasil. Estudos envolvendo o estabelecimento de valores de background geoquímico são importantes porque permitem avaliar riscos potenciais de contaminação e identificar concentrações naturais de elementos-traço de uma determinada área.

Palavras-chave: Background geoquímico, elementostraço, estudos ambientais, avaliação ambiental, contaminação.

\begin{abstract}
The establishment of background geochemical values as a relative measure to distinguish anthropogenic input from natural (geogenic and/or biogenic) concentrations of elements in different environmental samples, is one of the most important subjects in recent environmental sciences. The relationship between natural and anthropogenically altered concentrations of chemical species is a question that involves many implications in the geological, toxicological, biological and other areas of knowledge. This is especially important when geochemical interpretations of toxic elements are required. Thus, the principal objective of this article is to present and discuss the reasons for which the establishment of background geochemical values has implications in environmental studies. Throughout this article some concepts of the geochemical background used by various authors is presented and the main methods used for assessing background concentration of elements (direct and indirect methods). In addition, some examples of studies developed in Brazil are presented. Studies involving the establishment of background geochemical values are important because they allow the evaluation of potential contamination risks and the identification of natural trace-element concentrations in a determined area.
\end{abstract}

Keywords: Geochemical background, trace elements, environmental studies, environmental assessment, contamination. 


\section{Introdução}

De acordo com Galuszka (2007a), um dos assuntos mais importantes, nos estudos ambientais recentes, diz respeito ao estabelecimento de valores de background geoquímico para elementos e componentes orgânicos nos sistemas bióticos e abióticos. A relação entre as alterações naturais e as proporcionadas pela ação antrópica nas espécies químicas é uma questão que, segundo o autor, envolve implicações importantes nas áreas da geologia, toxicologia e biologia, bem como em outros campos do conhecimento. Isto é especialmente importante, quando análises geoquímicas de concentrações de elementos tóxicos são requeridas.

Vários estudos indicam uma elevação significativa na concentração de elementos-traço nos ecossistemas, após a revolução industrial (Villeneuve \& Holm, 1984; Richardson, 1992), sendo essa elevação muito bem documentada, sobretudo em sedimentos (Aston et al., 1973; Gschwend \& Hites, 1981; Baudo et al., 1990; Galuska \& Migaszewski, 2004). Diante disso, conforme exposto por Fadigas et al. (2006), é comum, durante a avaliação da extensão da poluição de uma área, comparar teores totais de elementos-traço obtidos desses locais com valores encontrados em condições naturais. Nesse caso, tais resultados são utilizados para calcular valores de background geoquímico, os quais permitem estabelecer padrões de qualidade ambiental, estudar o impacto de fontes de poluição antropogênicas ou, ainda, identificar fontes naturais de poluição, em uma determinada área (Reimann \& Garrett, 2005; Reimann et al., 2005; Galuszka, 2007a,b). Em caso de valores anômalos positivos, as influências antrópicas ocorrem, por definição, como contaminação (Matschullat, 2000a).

Conforme proposto por Matschullat et al. (2000a), background seria uma medida relativa usada para distinguir concentrações naturais de um dado elemento e a influência das atividades antrópicas nessas concentrações. Para os autores Reimann e Garrett (2005), Reimann et al. (2005) e Galuszka (2007a), o esta- belecimento de valores de background geoquímico de determinados elementos para um área é crucial, pois permite a separação das contribuições geogênicas/ biogênicas de um meio específico (águas, solos, sedimentos, plantas) daquelas de origem antrópica. Uma vez estabelecidos valores de background, para elementostraço, é possível a elaboração de mapas geoquímicos com enfoque geoambiental, considerados ferramentas importantes na gestão territorial e que podem ser transformados em guias de alerta aos gestores públicos, com relação à necessidade de remediação de locais onde são identificadas concentrações anômalas de elementos-traço (Costa, 2007).

Nessa casuística, têm sido utilizadas diferentes metodologias para se estabelecerem valores de background para elementos-traço, sendo que as mais comuns são: o uso de valores considerados normais (citados na literatura), a proposição de faixas de referência obtidas a partir de amostras-controle, tomadas em áreas sem atividade antrópica (Crock et al., 1992; Casarini, 2000), e a separação entre valores normais e anômalos, obtidos de uma coleção de dados, que inclui amostras contaminadas e não contaminadas (Wang, 1994; Tobias et al., 1997a, Tobias et al., 1997b).

Todavia, no Brasil, ainda não foi elaborado um sistema amplo contendo padrões de referência de elementos-traço, para a avaliação de áreas em relação à contaminação por esses elementos e poucos exemplos de trabalhos nacionais podem ser citados.

Dessa forma, o presente trabalho tem por objetivo apresentar as razões pelas quais o estabelecimento de valores de background geoquímico tem implicações em estudos ambientais. No decorrer do trabalho, são, primeiramente, discutidas a adoção e definições do termo background e, em seguida, é apresentada uma síntese de alguns dos principais métodos pelos quais os valores de background podem ser estabelecidos. Por fim, destacam-se alguns exemplos de trabalhos desenvolvidos, no Brasil, que utilizaram tais metodologias para estabelecer valores de background locais/ regionais.

\section{Definições de background geoquímico}

O termo "background geoquímico" foi, originalmente, introduzido em meados do século XX, para diferenciar a abundância de elementos nas formações rochosas mineralizadas e não-mineralizadas. Desde então, o termo tem sido utilizado em diferentes áreas das ciências ambientais, o que tem conduzido à ampliação de seu significado e à geração de variantes sem haver, no entanto, uma definição precisa.

Para Gough (1993), o significado mais comum do termo refere-se à escala de valores das concentrações de alguns elementos para um local influenciado por atividades antropogênicas. Todavia, conforme exposto por Plumlee (1999), em estudos de exploração geoquímica, o termo background se refere à concentração anômala de um elemento em um material sob investigação, tais como rochas, solos, plantas ou água.

Matschullat et al. (2000a) enfatizaram a ausência de uma definição clara sobre o termo e o definiram como sendo uma medida relativa a ser utilizada na distinção entre as concentrações naturais de um dado elemento e as concentrações influenciadas por atividades antrópicas. Segundo os autores, o estabelecimento de uma definição mais precisa para o termo é um passo importante que deve antecipar a sua utilização em qualquer estudo. Outra definição pode ser encontrada no estudo de Galuszka (2006), no qual background é definido como sendo uma concentração teoricamente natural de uma substância ou elemento em uma amostra, considerando as variáveis temporal e espacial da área sob investigação.

Todavia, o termo background geoquímico tem sido compreendido e utilizado de forma variada entre os estudiosos das áreas ambientais, fato este que tem suscitado uma discussão acerca das diversas definições dadas ao termo nas interfaces das ciências ambientais. A Tabela 1 sumariza outras definições propostas para o termo background. 
Aline Sueli de Lima Rodrigues et al.

Conforme é possível perceber, diferentes definições para o termo background têm sido adotadas em diferentes estudos ambientais. Uma breve análise dos significados apresentados na Tabela 1 conduz à conclusão de que os termos "background ambiental", "background antropogênico" e "background local" são sinônimos e que se referem às substâncias poluentes oriundas de fontes antropogênicas em escala regional não identificadas. Muitas vezes, a utilização dos termos acaba gerando confusão entre os estudiosos, haja vista as similaridades observadas nos seus significados e a discrepância entre as nomenclaturas adotadas.

Outros estudos têm utilizado, ainda, os termos "faixa de referência geoquímica" ou simplesmente "valores de referência" como sinônimos de background geoquímico, como é o caso do estudo pioneiro de Tidball et al. (1974) e, mais recentemente, o estudo de Nieto e Custodio (2005). Contudo é importante ressaltar que, independentemente do termo adotado em um estudo ambiental, o estabelecimento de valores referentes às concentrações naturais/ anômalas de determinados elementos traz, por si só, implicações significativas. Um exemplo disto é observado em estudos que têm por finalidade o monitoramento/investigação das interferências de uma atividade antrópica qualquer sobre o meio ambiente do entorno. Nesse caso, é importante distinguir o que seriam níveis normais de concentração de possíveis poluentes (ou seja, naturalmente presentes no meio ambiente) e níveis que, eventualmente, tenham sido adicionados pela atuação de tal atividade.

Tabela 1 - Diferentes definições do termo background.

\begin{tabular}{|c|c|c|}
\hline VARIANTES & DEFINIÇÕES & REFERÊNCIAS \\
\hline $\begin{array}{l}\text { Background } \\
\text { ambiental }\end{array}$ & $\begin{array}{c}\text { "Concentrações de substâncias inorgânicas naturais e de } \\
\text { substâncias inorgânicas de origem antrópica que são } \\
\text { representativas de uma dada região" }\end{array}$ & $\begin{array}{l}\text { Natural resources and } \\
\text { environmental protection } \\
\text { cabinet (2004) }\end{array}$ \\
\hline \multirow{2}{*}{$\begin{array}{l}\text { Background } \\
\text { antropogênico }\end{array}$} & $\begin{array}{l}\text { "Concentrações observadas tipicamente em uma dada região } \\
\text { como resultado das atividades humanas (não necessariamente } \\
\text { relacionadas a uma atividade de contaminação)" }\end{array}$ & Portier (2001) \\
\hline & $\begin{array}{l}\text { "Produtos químicos presentes no meio ambiente devido às } \\
\text { atividades humanas não relacionadas a fontes específicas de } \\
\text { liberação" }\end{array}$ & NFESC (2002) \\
\hline $\begin{array}{l}\text { Background } \\
\text { local }\end{array}$ & $\begin{array}{c}\text { "Concentrações de substâncias perigosas presentes no meio } \\
\text { ambiente, relacionadas à liberação por atividades } \\
\text { antropogênicas" }\end{array}$ & $\begin{array}{l}\text { Model Toxics Control Act - } \\
\text { Cleanup (2001) }\end{array}$ \\
\hline \multirow{2}{*}{$\begin{array}{l}\text { Background } \\
\text { natural }\end{array}$} & $\begin{array}{c}\text { "Quantidade de substâncias naturais no meio ambiente, isento } \\
\text { de fontes antropogênicas de poluição" }\end{array}$ & $\begin{array}{l}\text { Natural resources and } \\
\text { environmental protection } \\
\text { cabinet }(2004)\end{array}$ \\
\hline & $\begin{array}{l}\text { "Concentração de substâncias perigosas no meio ambiente não } \\
\text { relacionada à liberação por atividades antropogênicas" }\end{array}$ & $\begin{array}{l}\text { Model Toxics Control Act - } \\
\text { Cleanup (2001) }\end{array}$ \\
\hline $\begin{array}{l}\text { Background que } \\
\text { ocorre } \\
\text { naturalmente }\end{array}$ & $\begin{array}{l}\text { "Concentrações ambientais de elementos químicos presentes } \\
\text { no meio ambiente não influenciadas por atividades antrópicas" }\end{array}$ & NFESC (2002) \\
\hline $\begin{array}{l}\text { Background } \\
\text { pedogeoquímico }\end{array}$ & "Concentrações naturais de elementos presentes nos solos" & Baize \& Sterckeman (2001) \\
\hline $\begin{array}{l}\text { Background } \\
\text { pré-industrial }\end{array}$ & $\begin{array}{c}\text { "Concentrações de diferentes elementos de materiais coletados } \\
\text { e datados, de áreas estudadas no período pré-industrial" }\end{array}$ & $\begin{array}{l}\text { Matschullat et al. } \\
\qquad(2000 a)\end{array}$ \\
\hline
\end{tabular}


Conforme exposto no estudo conduzido por Fadigas et al. (2006), onde os autores analisaram as concentrações naturais de elementos-traço em algumas classes de solos brasileiros, o estabelecimento de valores de background geoquímico e de limites máximos considerados normais no solo tem sido feito através de diferentes métodos.

\section{Métodos utilizados na determinação de valores de background geoquímico}

Entre os métodos mais comumente utilizados, em estudos envolvendo a determinação de background geoquímicos, para elementos importantes, destacamse os métodos (i) direto (geoquímico) e (ii) indireto (estatístico), conforme discutido por Galuszka (2007b) e exposto, resumidamente, na Tabela 2.

\subsection{Método direto}

O método direto, também conhecido como método geoquímico, baseia-se na análise de amostras reconhecidamente isentas de interferência antrópica (Crommentuijn et al., 2000; Baize \& Sterckeman, 2001; Horckmans et al., 2005). Nesse caso, os valores de background são determinados, principalmente, por meio de valores médios da concentração de metais de uma área ou depósito sedimentar que não sofreu influência antropogênica. Conforme discutido por Costa (2007), a determinação de um dado valor de background, por esse método, requer um conhecimento sobre o comportamento geoquímico dos elementos estudados e sobre as condições ambientais prevalentes, incluindo o entendimento de condições paleoambientais e sedimentológicas, que nem sempre são facilmente entendidas.

Um exemplo da utilização de tal método pode ser observado no estudo conduzido por Kelley e Taylor (1997), no qual os autores coletaram amostras de águas naturais de áreas não impactadas e determinaram valores de $b a$ ckground para áreas do noroeste do Alaska. Já Kelley et al. (2003) utilizaram o método direto para investigar concentrações de cobre $(\mathrm{Cu})$ no nordeste do Chile.

\subsection{Método indireto}

Com relação ao método indireto, também chamado de método estatístico, ele exige o conhecimento específico de métodos exploratórios, que, de preferência, possuam baixa relação custo/área pesquisada. Como grande número de amostras geralmente é coletado, o custo das análises, para se obter a determinação exata da composição de cada amostra, seria elevado, fato que levou ao desenvolvimento de métodos semiquantitativos de rápida execução, com custo significativamente inferior. Conforme exposto por Frizzo et al. (2007), tais métodos, geralmente com extração parcial dos elementos, devem ter a característica essencial de reprodutibilidade dos resultados, embora possam perder em acurácia ou exatidão.

Assim, o método indireto tem, como característica intrínseca, a utilização de amostras estatísticas finitas, as quais constituem o todo ou parte da população amostrada que é efetivamente coletada e sobre as quais são efetuadas as medições requeridas (Frizzo et al., 2007). Através de procedimentos (ou de técnicas estatísticas) realizados por software computacionais, o conjunto de dados relativos a diferentes elementos químicos é analisado e, a partir disso, é possível interpretar tais dados e fazer considerações e inferências sobre a população amostrada e, conseqüentemente, sobre a área estudada.

Nos estudos de background geoquímico, várias técnicas estatísticas têm sido propostas. Entre elas destacam-se: análises de regressão linear (Selinus \& Esbensen, 1995), análise fractal (Li et al., 2003), normal probability plots (Tobias et al., 1997), representações boxplots, bem como o método de Lepeltier (Lepeltier, 1969), este último muito utilizado durante a década de 1970. Muitas dessas técnicas baseiam-se na construção de tabelas ou representações gráficas envolvendo amplitudes, freqüências e histogramas de concentrações dos

Tabela 2 - Diferentes métodos utilizados na determinação de valores de background geoquímico (Modificado de Galuszka, 2007b).

\begin{tabular}{c|c|c|c}
\hline MÉTODOS & ANÁLISES & $\begin{array}{c}\text { EXPRESSÃO DOS } \\
\text { VALORES DE } \\
\text { BACKGROUND }\end{array}$ & $\begin{array}{c}\text { PRINCIPAIS } \\
\text { REQUERIMENTOS }\end{array}$ \\
\hline $\begin{array}{c}\text { Direto } \\
\text { (Geoquímico) }\end{array}$ & $\begin{array}{c}\text { Requer o entendimento sobre o } \\
\text { comportamento geoquímico dos } \\
\text { elementos estudados }\end{array}$ & Média ou mediana & $\begin{array}{c}\text { Amostras não } \\
\text { influenciadas pela ação } \\
\text { antrópica }\end{array}$ \\
\hline $\begin{array}{c}\text { Indireto } \\
\text { (Estatístico) }\end{array}$ & $\begin{array}{c}\text { Análise de regressão linear, método } \\
\text { fractal, normal probability plots, } \\
\text { método de Lepeltier, etc. }\end{array}$ & $\begin{array}{c}\text { Valores } \\
\text { proporcionais }\end{array}$ & $\begin{array}{c}\text { Número de dados } \\
\text { maior que } 50\end{array}$ \\
\hline
\end{tabular}


Aline Sueli de Lima Rodrigues et al.

elementos encontrados nas amostras sob investigação. Conforme exposto nos estudos de Galuszka (2007a,b), praticamente todas as técnicas procuram identificar valores anômalos em uma série de dados estudada.

No que tange ao uso de freqüências acumuladas, também conhecidas por somas cumulativas, de acordo com o método gráfico proposto por Lepeltier (1969) e ligeiramente modificado por Matschullat et al. (2000a), o referido uso se baseia no pressuposto de que concentrações de elementos-traço possuem uma distribuição log-normal. Em outras palavras, abraça-se a idéia de que a distribuição de elementos-traço em materiais segue distribuição normal (ou de Gauss), com a particularidade de obedecê-la geralmente com valores expressos em logaritmos (Ahrens, 1954; Shaw, 1961).

Projetando-se as "somas cumulativas vs. concentração do elemento", em escala bilogarítmica, um desvio da distribuição log-normal pode ser percebido como uma inflexão na parte superior da curva, representando uma anomalia (Lepeltier, 1969; Matschullat et al., 2000a), conforme demonstrado na Figura 1. Nela, as duas setas apontam para dois momentos decisivos que separam os valores de background de um dado elemento (0 - 70\%) de amostras que possuem, naturalmente, concentrações elevadas do elemento (70 - 90\%) e de amostras que sofrem influência antropogênica $(>90-100 \%)$.

Esse procedimento foi desenvolvido para prospecção geoquímica de bens minerais, mas pode ser aplicado para determinar anomalias geoquímicas em diferentes amostras, conforme utilizado no estudo Luiz-Silva et al. (2006).

Outra abordagem estatística, freqüentemente utilizada em estudos envolvendo o estabelecimento de valores de background geoquímico, compreende a construção gráfica boxplot ou box-andwhisker, tomando-se, por base, a freqüência acumulada de um determinado elemento. Conforme descrito por Frizzo et al. (2007), através dos valores obtidos dessa freqüência, são identificados va- lores correspondentes a diferentes quartis $(25 \%, 50 \%$ e $75 \%)$ observados nas representações boxplot. Conforme ilustrado na Figura 2, os quartis $25 \%$ e $75 \%$ $\left(1^{\circ}\right.$ e $3^{\circ}$ respectivamente) formam os lados de um retângulo (box), seccionado pelo quartil $50 \%$, denominado mediana. As linhas (whiskers) se estendem de ambos os lados do box até os valores extremos ou, alternativamente, até os valores 1,5 vezes inferior e superior aos valores das amplitudes interquartis $25-50 \%$ e $50-75 \%$, respectivamente, e neste caso, resultados aquém e além das whiskers são considerados valores díspares, aberrantes e excessivos, conhecido como outliers ou, simplesmente, anômalos (Frizzo et al., 2007).

No Brasil, valores de background para o Estado de São Paulo foram definidos com base no quartil superior $(75 \%)$ dos resultados analíticos, conforme observados no estudo de Lemos (2001). Já, em solos da China, as concentrações de metais pesados em argilito foram usadas como padrões gerais (Wang, 1994).

É importante ressaltar que outros métodos estatísticos podem ser utilizados na análise e determinação de valores de background geoquímico conforme discutido de forma mais detalhada no estudo de Reimann et al. (2005). Entretanto, é necessário que se compreenda que apenas as análises estatísticas podem não ser suficientes para entender e definir as condições naturais que exis- tem em uma dada região, tampouco para a determinação exata e/ou inquestionável de valores de background geoquímico para determinados elementos químicos (Costa, 2007). Considera-se importante o entendimento dos processos geológicos, sedimentológicos e geoquímicos, que acabam por controlar a preponderância de concentrações naturais ou antrópicas dos elementos químicos em amostras de diferentes meios, sobretudo em sedimentos.

\section{Background geoquímico em estudos ambientais: exemplos de estudos desenvolvidos no Brasil}

Entender a dinâmica e a disponibilidade dos elementos-traço, bem como suas concentrações nos ecossistemas naturais, tem sido um grande desafio para muitos estudos envolvendo as questões ambientais, principalmente diante da constatação de que os impactos das atividades humanas sobre esses sistemas têm se intensificado nos últimos anos.

A industrialização, segundo Tarley e Arruda (2003), é uma das maiores dispersoras de elementos tóxicos, fato que, aliada ao rápido crescimento populacional e a algumas atividades agrícolas, au-

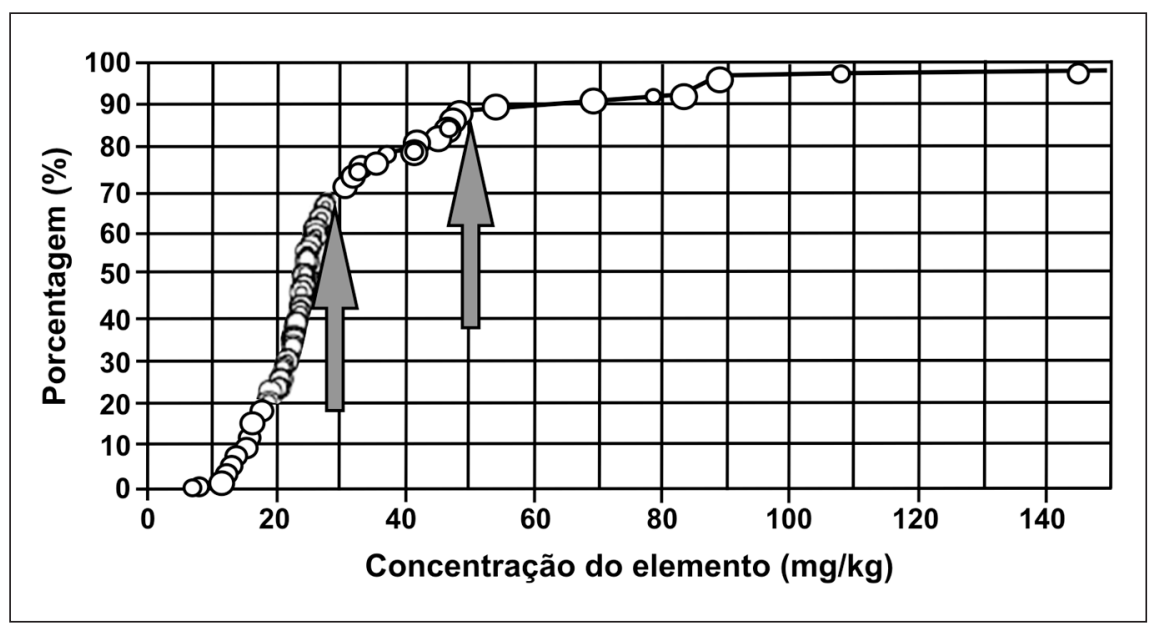

Figura 1 - Exemplo de projeção bilogarítmica da "soma cumulativa vs. Concentração" de um elemento $(\mathrm{mg} / \mathrm{kg})$. 
mentou o nível de poluição em diversos ambientes naturais (Santoyo et al., 2004), acarretando profundas transformações no meio ambiente. Além disso, as diversas emissões de sólidos, líquidos e de substâncias de variada natureza, oriundas de indústrias químicas, petroquímicas e/ou siderúrgicas, têm colocado tais atividades no cenário das grandes discussões ambientais mundiais. Bruno (2005) destaca, ainda, a liberação de elementos químicos através de partículas ou de vapores, resultantes, não somente da combustão de carvão, petróleo e gás natural, mas, também, da produção de cimento e de atividades metalúrgicas.

No que tange à liberação de elementos-traço, ela tem sido freqüentemente associada à contaminação e a efeitos tóxicos sobre diferentes comunidades bióticas presentes nos ecossistemas naturais. Embora alguns desses elementos sejam macronutrientes necessários aos seres vivos (Bruno, 2005), caso ultrapassem determinadas concentrações tornam-se perigosos, causam impactos negativos no ecossistema e podem, inclusive, ameaçar a saúde humana (Ribeiro Filho et al., 2001). As explorações, pelas grandes minerações, por exemplo, aumentam, significativamente, o teor de elementos tóxicos (principalmente As, $\mathrm{Cd}, \mathrm{Ba}, \mathrm{Cu}, \mathrm{Zn}$, Li e $\mathrm{Pb}$ ) no meio ambiente (Costa, 2007).

Nesse contexto, destaca-se a importância do estabelecimento de valores de background geoquímico nos estudos ambientais, seja através de pesquisas voltadas para a determinação de valores naturais de elementos-traço em diferentes amostras, seja para a constatação de valores anômalos prejudiciais às diversas formas de vida.

Sabe-se que, em ambientes aquáticos, as concentrações dos elementostraço têm sido, freqüentemente, associadas à litologia local (Bruno, 2005) e, em solos, há uma quantidade significativa de elementos-traço, que reflete, em maior ou menor grau, os teores do material de origem (Sumner, 1999). Já, em sedimentos, a concentração de elementostraço pode variar conforme a razão de

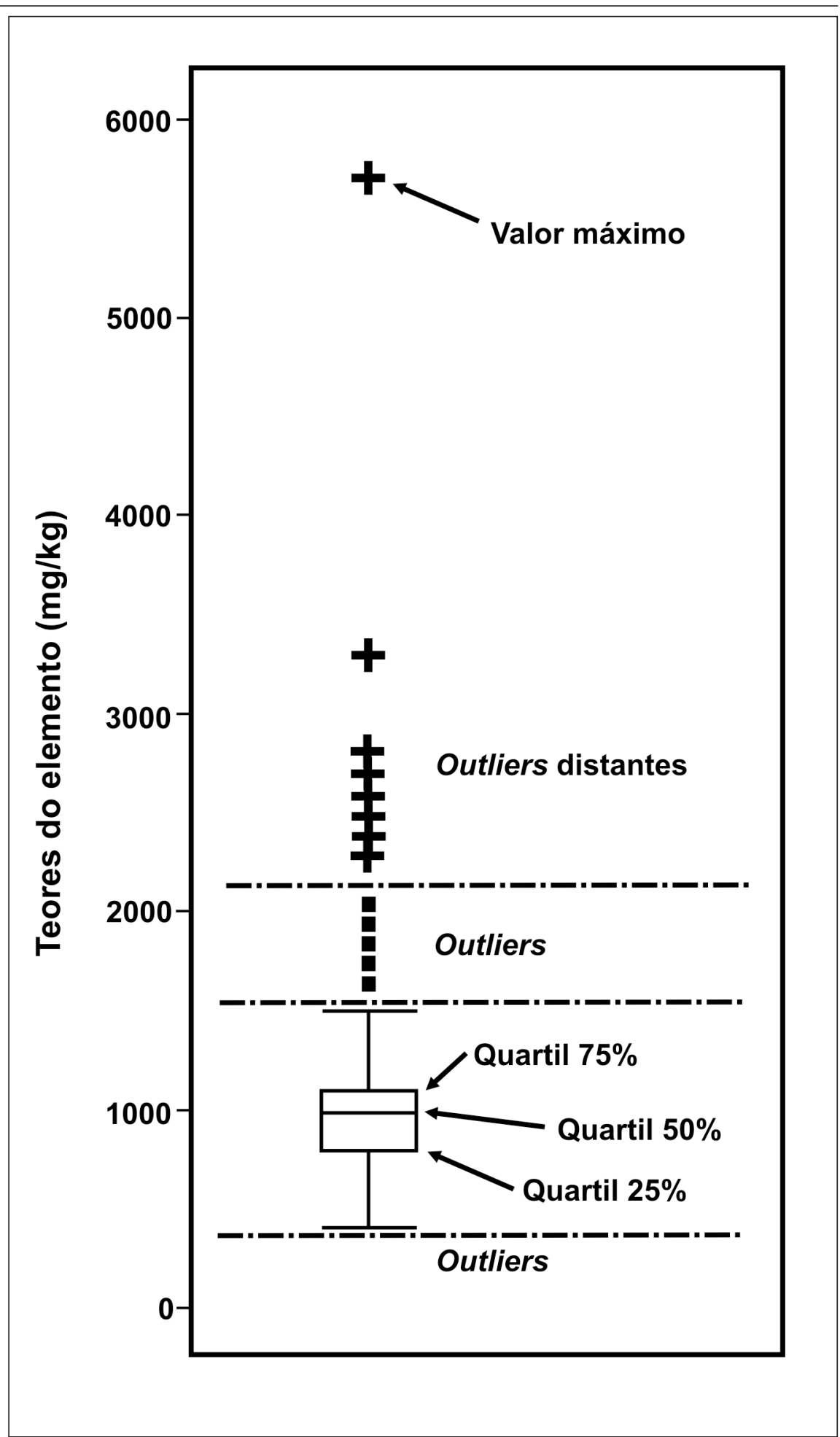

Figura 2 - Exemplo de boxplot para a concentração de um elemento.

sua deposição, sedimentação, natureza e tamanho das partículas, e conforme a presença ou não de matéria orgânica (Jesus et al., 2004). Contudo, quais seriam as reais contribuições das fontes naturais de liberação de elementos-traço aos ambientes que defrontam com as fontes antrópicas de poluição?

Conforme proposto pelo Naval Facilites Engineering Comand (NAVFAC) (NAVFAC, 2003) e exposto nos estudos de Matschullat et al. (2000b) 
Aline Sueli de Lima Rodrigues et al.

e Lemos (2000), o estabelecimento de valores de background para uma área consiste em uma ferramenta eficiente utilizada para quantificar o fator de enriquecimento de elementos tóxicos e quantificar a contaminação em uma região investigada, uma vez que as concentrações desses elementos podem variar em função de fatores geológicos e, também, em função de fatores antropogênicos.

No Brasil, conforme mencionado, poucos estudos têm sido desenvolvidos com o intuito de investigar as contribuições de diferentes fontes de liberação de elementos-traço nos ecossistemas, o que, de certa forma, propicia uma defasagem no conhecimento sobre essa temática. Todavia três estudos recentes merecem atenção, dada suas contribuições: Robaina et al. (2002), Fadigas et al. (2006) e Costa (2007).

\subsection{Metais pesados nos sedimentos de correntes, como indicadores de risco ambiental - vale do Rio dos Sinos, RS}

O estudo de Robaina et al. (2002), no qual se objetivou apresentar uma análise sobre a poluição por elementos-traço em sedimentos de corrente, foi realizado na região do vale do Rio dos Sinos, uma das regiões do Estado do Rio Grande do Sul com maior número de atividades industriais e com elevada ocupação urbana, abrangendo, principalmente, os municípios de Campo Bom, Novo Hamburgo e São Leopoldo.

A análise da poluição foi realizada através de etapas, entre as quais se destaca a avaliação da relação entre as concentrações de elementos-traço identificadas em amostras de argissolos e rochas e os valores de background estabelecidos no referido estudo, através do método indireto (estatístico). Os valores de background estabelecidos, para os elementos-traço importantes na região, são expressos na Tabela 3.
A partir desses resultados, os autores identificaram concentrações excessivas de elementos-traço em alguns tributários do rio Sinos, especialmente nos cursos d'água Luiz Rau, Pampa, Gauchinho e Kruze. Além disso, os elementostraço encontrados em maiores quantidades foram o $\mathrm{Cr}, \mathrm{Zn}, \mathrm{Cu}$ e $\mathrm{Pb}$, fato este que conduziu os autores a classificar tais cursos d'água como prioritários para trabalhos de fiscalização, controle e recuperação para qualquer tentativa de minimizar a poluição da região.

O estudo de Robaina et al. (2002) estabeleceu alguns valores de background para elementos-traço importantes na região do vale do Rio dos Sinos, servindo de base para estudos na região. Em adição, corrobora outros trabalhos que têm demonstrado a íntima associação existente entre os processos de urbanização/ industrialização e as altas concentrações de elementos-traço encontradas em uma dada bacia de drenagem.

\subsection{Proposição de valores de referência para a concentração natural de metais pesados em solos brasileiros}

Fadigas et al. (2006), considerando que os valores de background para identificação de elementos-traço não costumam levar em conta o tipo de solo inves- tigado, analisaram um conjunto de amostras de solos e propuseram, entre outras ações, o estabelecimento de valores de background, para alguns elementos-traço encontrados nos mais representativos solos brasileiros.

Para isso, os autores selecionaram, a princípio, as principais características físicas, químicas e mineralógicas dos solos capazes de influenciar os valores de background, entre elas os teores de argila, manganês, ferro e a capacidade de troca catiônica (CTC). A seguir, analisaram, por similaridade, um conjunto de 256 amostras de solos, correspondendo aos horizontes A, B ou C de 110 perfis de solos, dos quais $27 \%$ eram argilosos e $42 \%$ latossolos, as duas classes mais representativas de solos no Brasil. Além disso, amostras de cambissolos, luvissolos, nitossolos e planossolos também foram estudadas.

Em função das características dos solos, foram identificados 7 grupos (G1 a G7) de amostras das quais obtiveram as concentrações correspondentes ao quartil superior dos dados (75\%), sendo essas concentrações propostas como valores de background. Além disso, Fadigas et al. (2006) estabeleceram limites de tolerância para cada grupo, a partir da expressão anti-log $(m+2 s)$, cujos $m$ (média) e $s$ (desvio-padrão) dos dados foram transformados em logarítmicos na base 10. As Tabelas 4 e 5 apresentam, respectivamente, os valores de background

Tabela 3 - Valores de background propostos para os elementos $\mathrm{Pb}, \mathrm{Cu}, \mathrm{Zn}, \mathrm{Ni}, \mathrm{Cr}, \mathrm{Cd}$ em amostras de argissolos e rochas no estudo de Robaina et al., 2002.

\begin{tabular}{c|c|c|c}
\hline \multirow{2}{*}{ ELEMENTOS } & \multicolumn{3}{|c}{ CONCENTRAÇÃO (ppm) } \\
\cline { 2 - 4 } & $\begin{array}{c}\text { ROCHAS } \\
\text { SEDIMENTARES }\end{array}$ & ARGISSOLOS & $\begin{array}{c}\text { ROCHAS } \\
\text { VULCÂNICAS }\end{array}$ \\
\hline $\mathrm{Pb}$ & 36,08 & 51,81 & $\mathbf{1 0 , 0 0}$ \\
\hline $\mathrm{Cu}$ & 31,92 & 33,50 & $\mathbf{1 0 7 , 3 6}$ \\
\hline $\mathbf{Z n}$ & 77,65 & 81,32 & $\mathbf{1 1 6 , 5 2}$ \\
\hline $\mathbf{N i}$ & 45,79 & 49,29 & $\mathbf{1 2 8 , 0 0}$ \\
\hline $\mathrm{Cr}$ & 74,17 & 81,20 & $\mathbf{1 5 4 , 0 4}$ \\
\hline $\mathrm{Cd}$ & $\mathbf{0 , 5 4}$ & $\mathbf{0 , 5 4}$ & - \\
\hline
\end{tabular}


Valores de background geoquímico e suas implicações em estudos ambientais

e os limites de tolerância estabelecidos para os elementos $\mathrm{Cd}, \mathrm{Co}, \mathrm{Cr}, \mathrm{Cu}, \mathrm{Ni}, \mathrm{Pb}$, Zn.

Para os autores, o estabelecimento dos valores de background, para os elementos $\mathrm{Cd}, \mathrm{Co}, \mathrm{Cr}, \mathrm{Cu}, \mathrm{Ni}, \mathrm{Pb}$ e $\mathrm{Zn}$, pode ser utilizado em avaliações preliminares de áreas com suspeita de poluição ou com uso agrícola, cujos solos possuam características similares às dos solos estudados em questão. Em outras palavras, os autores afirmam que é possível avaliar solos sob atividade antrópica e saber se há motivo para suspeitar de que a área esteja poluída. Alguns tipos de solos brasileiros têm propriedades específicas, que fazem com que a concentração de determinados elementos-traço seja, naturalmente, mais elevada, sem que isso chegue a ser prejudicial.

\subsection{Registro histórico de contaminação por metais pesados, associada à exploração aurífera da bacia do Ribeirão do Carmo, Quadrilátero Ferrífero}

O estudo de Costa (2007) foi desenvolvido na região do Quadrilátero Ferrífero (QF) - MG, considerada uma das regiões mais importantes de exploração aurífera do Brasil. Na ocasião, a autora estudou, entre outros itens, a caracterização da influência da exploração aurífera histórica (mineração e garimpo) na composição geoquímica dos sedimentos de planícies de inundação e terraços aluviais na bacia do Ribeirão do Carmo (Alto Rio Doce), considerados sistemas coletores e acumuladores de elementos químicos de elevada toxicidade. Para tal, foram analisados sedimentos coletados em vários trechos ao longo do ribeirão do Carmo e valores de background para elementos-traço importantes na região foram propostos. Posteriormente, com base nesses valores estabelecidos, nos valores médios da crosta continental superior, bem como nos valores de guias de qualidade de sedimentos, a contaminação na área foi avaliada de forma quantitativa.

Tabela 4 - Valores considerados normais para o teor de $\mathrm{Cd}, \mathrm{Co}, \mathrm{Cr}, \mathrm{Cu}, \mathrm{Ni}, \mathrm{Pb}$ e $\mathrm{Zn}$ em solos não poluídos, propostos como valores de background ${ }^{1}$ por Fadigas et al. (2006).

\begin{tabular}{|c|c|c|c|c|c|c|c|}
\hline \multirow{3}{*}{ GRUPOS } & \multicolumn{7}{|c|}{ ELEMENTOS } \\
\hline & \multicolumn{7}{|c|}{ Concentração no solo (mg/kg) } \\
\hline & $\mathrm{Cr}$ & Co & $\mathbf{N i}$ & $\mathrm{Cu}$ & $\mathrm{Zn}$ & Cd & $\mathbf{P b}$ \\
\hline 1 & 55 & 29 & 35 & 119 & 79 & 1 & 19 \\
\hline 2 & 48 & 10 & 18 & 19 & 44 & 0,8 & 25 \\
\hline 3 & 65 & 4 & 25 & 16 & 23 & 1,6 & 16 \\
\hline 4 & 35 & 10 & 17 & 12 & 35 & 0,9 & 18 \\
\hline 5 & 23 & 4 & 7 & 6 & 12 & 0,4 & 22 \\
\hline 6 & 43 & 2 & 12 & 2 & 12 & 0,4 & 3 \\
\hline 7 & 19 & 2 & 5 & 3 & 6 & 0,3 & 40 \\
\hline$Q S m^{2}$ & 41 & 8 & 17 & 25 & 30 & 0,8 & 20 \\
\hline
\end{tabular}

${ }^{1}$ Concentração considerada normal para os solos pertencentes a cada grupo e que corresponde ao valor do quartil superior (75\%) da distribuição de freqüência dos dados amostrais, em cada grupo.

${ }^{2}$ Quartil superior médio entre os grupos.

Tabela 5 - Limites de tolerância para os elementos $\mathrm{Cd}, \mathrm{Co}, \mathrm{Cr}, \mathrm{Cu}, \mathrm{Ni}, \mathrm{Pb}$ e $\mathrm{Zn}$ propostos por Fadigas et al. (2006).

\begin{tabular}{|c|c|c|c|c|c|c|c|}
\hline \multirow{3}{*}{ GRUPOS } & \multicolumn{7}{|c|}{ ELEMENTOS } \\
\hline & \multicolumn{7}{|c|}{ Concentração no solo (mg/kg) } \\
\hline & $\mathrm{Cr}$ & Co & $\mathrm{Ni}$ & $\mathrm{Cu}$ & Zn & Cd & $\mathbf{P b}$ \\
\hline 1 & 114 & 44 & 92 & 283 & 149 & 2 & 35 \\
\hline 2 & 94 & 34 & 45 & 41 & 92 & 2 & 66 \\
\hline 3 & 131 & 12 & 46 & 48 & 42 & 4 & 31 \\
\hline 4 & 81 & 21 & 43 & 41 & 65 & 2 & 60 \\
\hline 5 & 61 & 9 & 21 & 11 & 24 & 1 & 84 \\
\hline 6 & 53 & 3 & 19 & 2 & 14 & 1 & 4 \\
\hline 7 & 41 & 8 & 10 & 5 & 13 & 1 & 154 \\
\hline
\end{tabular}

No que tange à análise de valores de background, as concentrações propostas pelos métodos direto (geoquímico) e indireto (estatístico), para os elementos $\mathrm{As}, \mathrm{Cu}, \mathrm{Zn}, \mathrm{Ni}, \mathrm{Cr}$, Ba e Mn, provenientes das áreas que foram intensamente afetadas pela exploração de ouro no passado, são sumarizadas na Tabela 6.

No que tange à avaliação quantitativa da contaminação antropogênica na região estudada, Costa (2007), comparando seus resultados com os valores estabelecidos pela CETESB para o Esta- 
Aline Sueli de Lima Rodrigues et al.

do de São Paulo (Lemos, 2001), observou que a região apresenta valores de background, para elementos-traço (por exemplo, As e Cd) em sedimentos que excedem os limites considerados seguros para a saúde humana. Esse fato reflete as concentrações alarmantes desses elementos nos sedimentos, embora naturais, indicando risco à saúde humana e a necessidade de intervenção na área de forma a interceptar as vias de exposição.

\section{Considerações finais}

Conforme exposto, o estabelecimento de valores de background geoquímico para elementos-traço tem significativas implicações nos estudos ambientais, sobretudo naqueles ligados à avaliação de áreas contaminadas, ou que estão sob risco potencial de poluição.

$\mathrm{O}$ interesse pela quantificação da poluição por elementos-traço, em diferentes sistemas naturais, traz, como conseqüência direta, o levantamento de dados que pode auxiliar na avaliação de impactos ambientais e de suas complexas relações com as atividades econômicas. Além disto, auxilia na identificação das possíveis fontes de poluição, bem como na atribuição de tendências nas mudanças de comportamento na estruturação industrial e na implementação de tecnologias de controle da poluição.

É consenso que os ecossistemas naturais vêm sendo depositários de uma variedade de subprodutos, provenientes das atividades antrópicas, e que a presença de elementos, potencialmente tóxicos, é responsável por efeitos adversos no ambiente, com repercussão na economia e na saúde pública. Entretanto é imprescindível a distinção das contribuições das fontes de poluição por elementos-traço, principalmente diante do fato de que existem fontes naturais desses elementos.

Conforme discutido por Fadigas et al. (2006), elevados teores de elementostraços podem ser encontrados em amostras de solos, sem que, necessariamente, eles estejam ligados às atividades antrópicas, podendo estar, em alguns casos, ligados a fatores geogênicos/natu- rais, conforme é o caso de solos basálticos, que, naturalmente, apresentam elevadas concentrações de elementos-traço (como, por exemplo, latossolos e nitossolos vermelhos distroféricos).

Ressalta-se que análises por meio do estabelecimento de valores de background podem ser úteis no fornecimento de informações que facilitam a interpretação de resultados obtidos de uma área suspeita de contaminação, principalmente por levar em consideração a existência de propriedades específicas de amostras que fazem com que o teor dos elementos-traço seja naturalmente mais elevado. Além disso, tais análises podem ser um excelente critério para julgar a extensão de acumulação de um determinado metal no solo, o que pode orientar os estudos da potencialidade de ocorrência de problemas nutricionais (em plantas e animais), de saúde humana e ambientais. Se não há o conhecimento de qual é o nível considerado natural de um determinado elemento no ecossistema, como saber se ele foi antropicamente contaminado ou se ele possui uma concentração que pode afetar os seres vivos?

Tabela 6 - Valores de background propostos para elementos-traço provenientes de sedimentos da bacia do Ribeirão do Carmo (Costa, 2007).

\begin{tabular}{|c|c|c|c|c|c|c|c|c|}
\hline \multirow{3}{*}{$\begin{array}{l}\text { ELEMENTOS } \\
(\mathrm{mg} / \mathrm{kg})\end{array}$} & \multirow{2}{*}{\multicolumn{2}{|c|}{$\begin{array}{c}\text { MÉTODO DIRETO } \\
\text { (GEOQUÍMICO) }\end{array}$}} & \multicolumn{6}{|c|}{ MÉTODO INDIRETO (ESTATÍSTICO) } \\
\hline & & & \multicolumn{2}{|c|}{$n=120$} & \multicolumn{2}{|c|}{$\begin{array}{l}\text { Fácies argilosas } \\
\qquad(n=62)\end{array}$} & \multicolumn{2}{|c|}{$\begin{array}{l}\text { Fácies cascalho } \\
\qquad(\mathrm{n}=84)\end{array}$} \\
\hline & Média & Máximo & Média & Máximo & Média & Máximo & Média & Máximo \\
\hline As & 2,00 & 10,00 & 35,30 & 810,00 & 10,21 & 241,40 & 229,30 & 705,00 \\
\hline $\mathrm{Cu}$ & 44,00 & 188,00 & 135,10 & - & 188,80 & - & 114,90 & - \\
\hline $\mathrm{Zn}$ & 68,00 & 513,00 & 124,00 & - & 118,90 & - & 39,00 & 124,00 \\
\hline Cd & 13,30 & 35,80 & 14,06 & - & 14,06 & - & 1,15 & - \\
\hline $\mathrm{Ni}$ & 18,00 & 104,00 & 53,10 & - & 12,02 & 50,10 & 104,40 & - \\
\hline $\mathrm{Cr}$ & 63,00 & 198,00 & 111,00 & - & 111,00 & - & 139,80 & - \\
\hline $\mathrm{Ba}$ & 25,00 & 130,00 & 18,50 & - & 18,11 & 196,10 & 51,90 & 655,00 \\
\hline Mn & 218,00 & 1911,00 & 189,50 & 5800,00 & 189,68 & - & 398,10 & 1694,60 \\
\hline
\end{tabular}


Por fim, é necessária a sensibilização das autoridades competentes para que os órgãos de fomento de Ciência \& Tecnologia estaduais e federais possam criar linhas de pesquisa específicas para subsidiar estudos que visem a propor valores de background geoquímico para elementos-traço importantes. Na medida em que estudos envolvendo essa temática forem sendo desenvolvidos, para os mais variados sistemas naturais, é necessária a divulgação dos mesmos no sentido de serem utilizados como fontes de informações e guia para estudos futuros.

\section{Referências bibliográficas}

AHRENS, L.H. The log-normal distribution of the elements (A fundamental law of geochemistry and its subsidiary). Geochimica et Cosmochimica Acta, v. 5, p. 49-73, 1954.

ASTON, S.R., BRUTY, D., CHESTER, R., PADGHAM, R.C. Mercury in lake sediments: A possible indicator of technological growth. Nature, v. 241, p. 450-451, 1973.

BAIZE, D., STERCKEMAN, T. Of the necessity of knowledge of the natural pedogeochemical background content in the evaluation of the contamination of soils by trace elements. Science of the Total Environment, v. 264, p. 127-139, 2001.

BAUDO, R., GIESY, J.P., MUNTAU, H. Sediments: chemistry and toxicity of inplace pollutants. Boston: Lewis Publishers, Ann Arbor, MI, 1990. 424p.

BRUNO, A.P.S. Concentração de metais pesados e elementos-traço em dois trechos da planície de inundação do baixo curso rio Jaboatão, Pernambuco, Brasil. Programa de Pós-Graduação em Geociências do Centro de Tecnologia e Geociências da Universidade Federal de Pernambuco, 2005. 66p. (Dissertação de Mestrado).

CASARINI, D.C.P. Proposta de valores de referência de qualidade eintervenção para solos e águas subterrâneas no estado de São Paulo. In: SEMINÁRIO INTERNACIONAL SOBRE QUALIDADE DE SOLOS E ÁGUAS SUBTERRÂNEAS, 2. Anais... São Paulo: CETESB, 2000. 165p.

COSTA, A.T. Registro histórico de contaminação por metais pesados, associadas à exploração aurífera no alto e médio curso da bacia do Ribeirão do Carmo, QF: um estudo de sedimentos de planícies de inundação e terraços aluviais. Programa de Pós-Graduação em Evolução Crustal e Recursos Naturais, Departamento de Geologia, Universidade Federal de Ouro Preto, 2007. 257p. (Tese de Doutorado).

CROCK, J.G., SEVERSON, R.C., GOUGH, L.P. Determining baselines and variability of elements in plants and soils near the Kenai National Wildlife Refuge Alaska. Water, Air, and Soil Pollution, Netherlands, v. 63 , n. $3 / 4$, p. 253-271, 1992.

CROMMENTUIJN, T., SIJM, D., de BRUIJN, J., VAN DEN HOOP, M., VANLEEUWEN, K., VAN DE PLASSCHE, E. Maximum permissible and negligible concentrations for metals and metalloids in the Netherlands, taking into account background concentrations. Journal Environmental Management, v. 60, p. 121-143, 2000.

FADIGAS, F. de S. et al. Proposição de valores de referência para a concentração natural de metais pesados em solos brasileiros. Revista Brasileira de Engenharia Agrícola e Ambiental, v. 10, n.3, p. 699-705, 2006.

FRIZZO, S.J., LICHT, O.A.B. Estatística uni e bivariadas aplicadas à prospecção geoquímica. In: LICHT, O.A.B., DEMELLO, C.S.B., DA-SILVA, C.R. (eds). Prospecção geoquímica de depósitos minerais metálicos, não-metálicos, óleo e gás. Rio de Janeiro: Sociedade Brasileira de Geoquímica (SBGq) e Serviço Geológico do Brasil (CPRM), 2007. 788p.

GALUSZKA, A., MIGASZEWSKI, Z.M. Concentrations of polynuclear aromatic hydrocarbons in sediments of Lake Wigry and selected rivers near Suwa ${ }^{3} \mathrm{ki}$, northeastern Poland. Prace Komisji Paleogeografii Czwartorze $e^{2}$ u Polska Akademia Umieje'tnos'ci (in Polish with English summary), v. 2, p. 49-54, 2004.

GALUSZKA, A. Methods of determining geochemical background in environmental studies. Problems of landscape ecology. Polish association of landscape ecology - Warsaw (in Polish with English summary), v. 16, n. 1, p. 507-519, 2006.

GALUSZKA, A. A review of geochemical background concepts and an example using data from Poland. Environmental Geology, v. 52, p. 861-870, 2007a.

GALUSZKA, A. Different approaches in using and understanding the term "Geochemical Backgroun"- pratical implications for environmental studies. Polish Journal of Environmental Studies, v. 16, n. 3, p. 389-395, 2007 b.

GOUGH, L.P. Understanding our fragile environment. Lessons from geochemical studies. United States Geological Survey Circular, v. 1105, p. 1-34, 1993.

GSCHWEND, P.M., HITES, R.A. Fluxes of polycyclic aromatic hydrocarbons to marine and lacustrine sediments in the northeastern US. Geochimica Cosmochimica Acta, v. 45, p. 2359-2367, 1981.

HORCKMANS, L., SWENNEN, R., DECKERS, J., MAQUIL, R. Local background concentrations of trace elements in soils: a case study in the Grand Duchy of Luxemburg. Catena, v. 59, p. 279-304, 2005.

JESUS, H.C., COSTA, E.A., MENDONÇA, A.S.F., ZANDONADE, E. Distribuição de metais pesados em sedimentos do sistema estuarino da ilha de Vitória do Espírito Santo, ES. Química Nova, v. 27, n. 3, p. 378-386, 2004.

KELLEY, K.D., TAYLOR, C.D. Environmental geochemistry of shalehosted $\mathrm{Ag}-\mathrm{Pb}-\mathrm{Zn}$ massive sulfide deposits in northwest Alaska: natural background concentrations of metals in water from mineralized areas. Applied Geochemistry, v. 12, p. 397-409, 1997.

KELLEY, D.I., HALL, G.E.M., CLASS, G., HAMILTON, I.C., MCEWEN, R.M. The use of partial extraction geochemistry for copper exploration in northern Chile. Geochemistry: Exploration, Environment, Analysis, v. 3, p. 85-104, 2003.

LEMOS, M.M.G. Metodologia adotada para o estabelecimento dos valores de referência de qualidade para solos e águas subterrâneas no Estado de São Paulo. In: Prevenção e controle da poluição do solo e das águas subterrâneas. São Paulo: CETESB, 2000. p. 68-77.

LEMOS, M.M.G. Relatório de estabelecimento de valores orientadores para solos e águas subterrâneas no estado de São Paulo. São Paulo: CETESB, 2001. p. 2001.73 p.

LEPELTIER, C. A simplified treatment of geochemical data by graphical representation. Economic Geology, v. 64, p. 538-550, 1969.

LI, C.H., MA, T., JUNFA, S. Application of a fractal method relating concentrations and distances for separation of geochemical anomalies from background. Journal of Geochemical Exploration, v. 77, p. 167-175, 2003. 
Aline Sueli de Lima Rodrigues et al.

LUIZ-SILVA, W., MATOS, R.H.R., KRISTOSCH, G.C., MACHADO, W. Variabilidade espacial e sazonal da concentração de elementos-traço em sedimentos do sistema estuarino de Santos-Cubatão (SP). Química Nova, v. 29, n. 2, p. 256-263, 2006.

MATSCHULLAT, J., OTTENSTEIN, R., REIMANN, C. Geochemical background - can we calculate it? Environmental Geology, v. 39, p. 990-1000, 2000a.

MATSCHULLAT, J., BORBA, R.P., DESCHAMPS, E., FIGUEIREDO, B.F., GABRIO, T., SCHWENK, M. Human and environmental contamination in the Iron Quadrangle, Brazil. Applied Geochemistry, v.15, p.181-190, 2000b.

MODEL TOXICS CONTROL ACT Cleanup - Departament od ecology. Washington Administrative Code. 173 340-200, 2001 (www.leg.wa.gov.wac).

NATURAL RESOURCES AND ENVIRONMENTAL PROTECTION CABINET. Kentucky guidance for ambient background assessment [citado em 2004]. Disponível em: http:// ra i s.orn l.gov/hom e page/ AmbientBackgroundAssessment.pdf. Acessado em: 31 out. 2008.

NAVAL FACILITIES ENGINEERING COMMAND (NFESC). Guidance for environmental background analysis, Volume 1: Soil. Washington: NFESC, 2002. 188p.

NIETO, P., CUSTODIO, E., MANZANO, M. Baseline groundwater quality: a European approach. Environmental Science and Policy, v. 8, p. 399-409, 2005.

PLUMLEE, G.S. The environmental geology of mineral deposits. In: PLUMLEE, G.S., LOGSDON, J.J. (eds.). The environmental geochemistry of mineral deposits. Part A, Processes, Techniques, and Health Issues. Society of Economic Geologists Reviews in Economic Geology, v. 6A, p. 71-116, 1999.

PORTIER, K.M. Statistical issues in assessing anthropogenic background for arsenic. Environmental Forensics, v. 2, p. 155-160, 2001.

REIMANN, C., GARRET, R.G. Geochemical background - concept and reality. Science of the Total Environment, v. 350, p. 12-27, 2005.

REIMANN, C., FILZMOSER, P., GARRETT, R.G., Background and threshold: critical comparison of methods of determination. Science of the Total Environment, v. 346, p. 1$16,2005$.

RIBEIRO-FILHO, M.R., SIQUEIRA, J.O., CURI, N., SIMÃO, J.B.P. Fracionamento e biodisponibilidade de metais pesados em solo contaminado, incubado com materiais orgânicos e inorgânicos. Revista Brasileira de Ciências do Solo, v. 25, p.495-507, 2001.

RICHARDSON, D.H.S. Pollution monitoring with lichens. Slough, England: Richmond Publishing Co. Ltd. 76p. 1992.

ROBAINA, L.E.S., FORMOSO, M.L.L., PIRES, C.A.F. Metais pesados nos sedimentos de corrente como indicadores de risco ambiental - vale do Rio dos Sinos, RS. Revista do Instituto Geológico, v. 23, n. 2, p. 35-47, 2002.

SANTOYO, E., SANTOYO-GUTIÉRREZ, S.E., VERMA, S.P. Trace analysis of heavy metals in groundwater samples by ion chromatography with post-column reaction and ultraviolet-visible detection. Journal of Chromatography, v. 884, n. 2, p. 229-241, 2000.

SELINUS, O.S., ESBENSEN, K. Separating anthropogenic from natural anomalies in environmental geochemistry. Journal of Geochemical Exploration, v. 55, p. 55-66, 1995.

SHAW, D.M. Element distribution laws of geochemistry. Geochimica et Cosmochimica Acta, v. 23, p. 116-134, 1961.

SUMNER, M.E. Handbook of soil science. Washington: Press. 631p.

TARLEY CRT., ARRUDA MAZ. Adsorventes naturais: potencialidades e aplicações da esponja natural (Luffa cylindrica) na remoção de chumbo em efluentes de laboratório. Revista Analytica, v. 4, p. 25-31, 2003.

TIDBALL, R.R., ERDMAN, J.A., EBENS, R.J. Geochemical baselines for sagebrush and soil. Powder River Basin. Montana - Wyoming. United States Geological Survey OpenFile Report, v. 74, p. 6-13, 1974.

TOBIAS, F.J., BECH, J., ALGARRA, P.S. Establishment of the background levels of some trace elements in soils of NE Spain with probability plots. Science of the Total Environment, v. 206, p. 255-265, 1997a.

TOBIAS, F.J., BECH, J., ALGARRA, P.S. Statistical approach to discriminate background and anthropogenic input of trace elements in soils of Catalonia, Spain. Water, Air, and Soil Pollution, Netherlands, v. 100, p. 63-78, $1997 \mathrm{~b}$.

VILLENEUVE, J.P., HOLM, E. Atmospheric background of chlorinated hydrocarbons studied in Swedish lichens. Hemosphere, v. 13, p. 1133-1138, 1984.

WANG, H.K. Research methodology for determining background levels of trace elements in Chinese soils. Environmental Geochemistry and Health, special issue, p.397-413, 1994.

Artigo recebido em 01/11/2008 e aprovado em 19/03/2009.

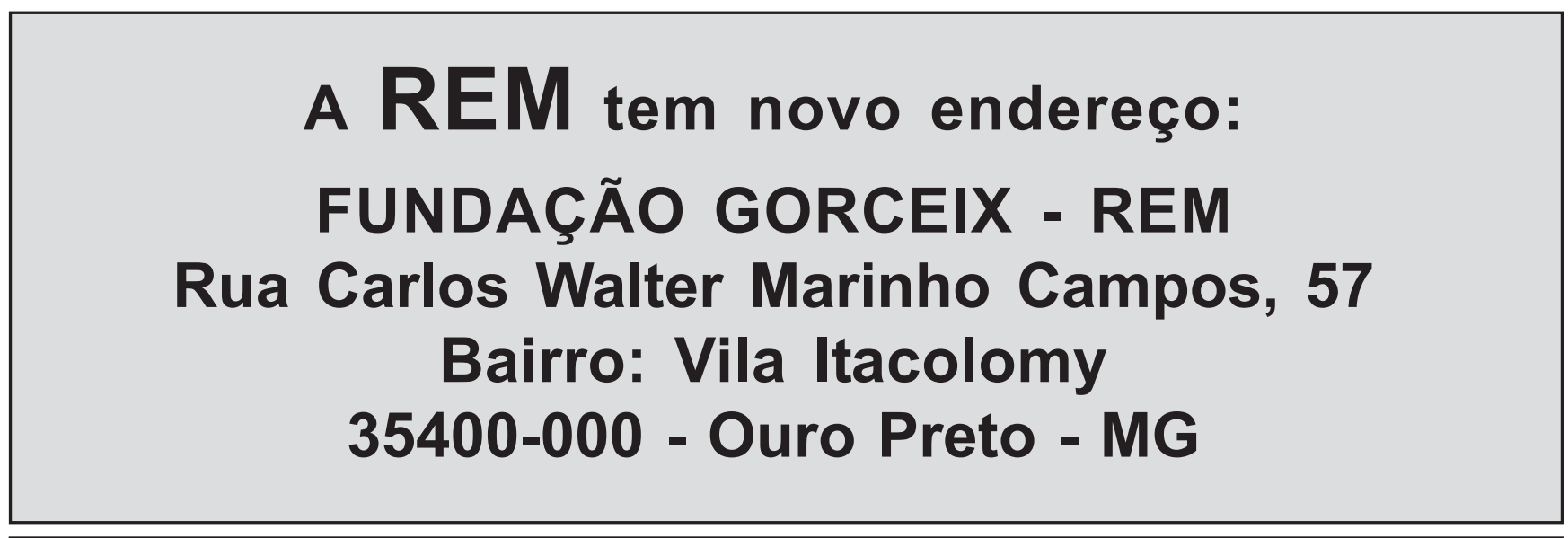

